\title{
Novedades corológicas de Taraxacum F.H. Wigg. (Asteraceae) para la flora de Argentina, Colombia y Perú
}

\author{
Antonio Galán de Mera', Eliana Linares Perea ${ }^{2}$, Juan Montoya Quino ${ }^{3}$, Iván Torres Marquina ${ }^{4}$ \& José \\ Alfredo Vicente Orellana ${ }^{1}$ \\ 'Laboratorio de Botánica, Facultad de Farmacia, Universidad CEU San Pablo, apartado 67, 28660 Boadilla del Monte, \\ Madrid, España. \\ Estudios Fitogeográficos del Perú, Sánchez Cerro 219, Manuel Prado, Paucarpata, Arequipa, Perú. \\ ${ }^{3}$ Herbario CPUN, Departamento de Biología, Universidad Nacional de Cajamarca, Cajamarca, Perú. \\ ${ }^{4}$ Laboratorio de Botánica, Facultad de Ciencias de la Salud (Farmacia), Universidad Privada Antonio Guillermo Urrelo \\ (UPAGU), Jr. José Sabogal 913, Cajamarca, Perú.
}

\section{Correspondencia}

A. Galán de Mera

e-mail: agalmer@ceu.es

Recibido: 6 noviembre 2017

Aceptado: 13 febrero 2018

Publicado on-line: mayo 2018

\author{
Chorological novelties of Taraxacum F.H. Wigg. (Asteraceae) for the flora of \\ Argentina, Colombia and Peru
}

Palabras clave: Taraxacum, América del Sur, distribución, fitosociología.

Key words: Taraxacum, South America, distribution, phytosociology.
El género Taraxacum se distribuye por todo el hemisferio norte y algunas zonas de América Central y del Sur (Cordillera Andina, S de Brasil y Tierra del Fuego), islas Galápagos (Ecuador), islas Juan Fernández (Chile), Australia y Nueva Zelanda (Galán de Mera, 2017). Comprende unas 100 especies sexuales y posiblemente más de 1500 microespecies agámicas repartidas en al menos 60 secciones, de las cuales 6 están representadas en América del Sur (Uhlemann et al., 2004). Su sistemática es problemática pues reúne plantas con tres tipos de reproducción: alogamia, autogamia (rara) y apomixis (basada en diplosporia meiótica) (Van Baarlen et al., 2000). Las especies apomícticas pueden producir polen fértil y cruzarse con diploides o poliploides produciendo nuevos linajes hibridógenos que dan lugar a plantas con una morfología muy variada de difícil identificación (Vašut, 2003), aunque con una cierta constancia en sus caracteres, como la estructura y color de las hojas, la posición, morfología y coloración de las brácteas externas del involucro, o la estructura y color de los aquenios.

Actualmente, en América del Sur, no existe un tratamiento homogéneo para el género. Así, en Perú, no se acepta másque el nombre de T. officinale F.H. Wigg. (Brako \& Zarucchi, 1993; Beltrán, 2016) a pesar de un artículo previo para la flora peruana (Galán de Mera \& Linares Perea, 2008), y aunque ya otros autores como Herrera (1941), basándose en la obra de Handel-Mazzetti (1907),

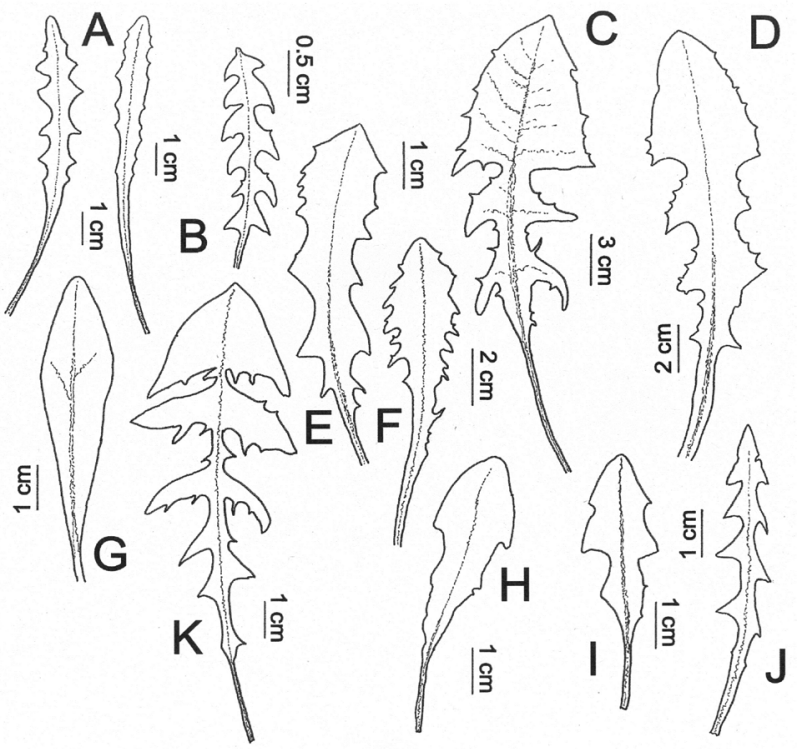

Figura 1. Silueta de las hojas de las especies de Taraxacum estudiadas. A: T. gilliesii [E. Linares \& A. Galán 4547c (USP 3399) y 4556 (USP 3400)]. B: T. plumbeum [E. Linares \& A. Galán 4524d (USP 3398)], C: T. stictophyllum [E. Linares \& A. Galán 4522d (USP 3379)], D: T. duplidentifrons [E. Linares \& A. Galán 4543 (USP 3381)]. E y F: Taraxacum fernandezianum [E. Linares \& A. Galán 4527 (CPUN)]. G, H e I: T. spathulatum [E. Linares \& A. Galán 4535c (USP 3393)], J: T. argutifrons [O. Rangel, J. Pinto \& A. Galán 4549 (USP 3405)], K: T. craspedotoides [O. Rangel, J. Pinto \& A. Galán 4552 (USP 3395)].

Figure 1. Outline of leaves of the studied species of Taraxacum. 

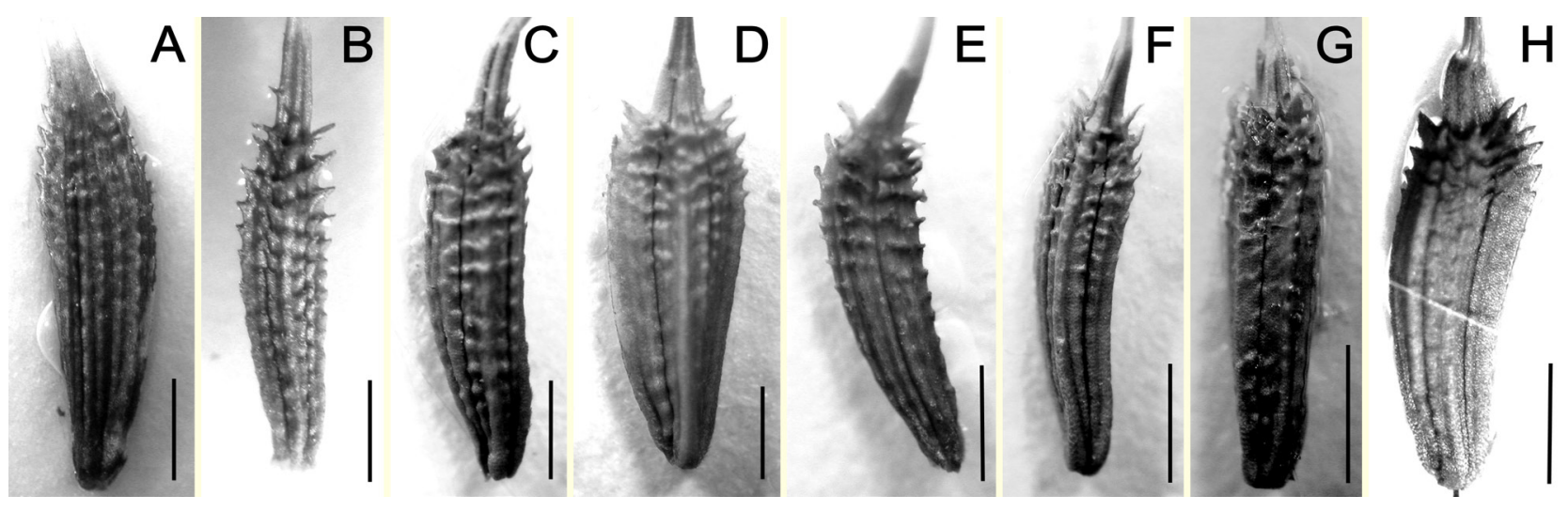

Figura 2. Aquenios de las especies de Taraxacum estudiadas (barras $=1 \mathrm{~mm}$ ). A:T. gilliesii [E. Linares \& A. Galán 4547c (USP 3399)], B: T. plumbeum [E. Linares \& A. Galán 4525(USP 3397)], C: T. stictophyllum [E. Linares \& A. Galán 4523 (USP 3380)], D: T. duplidentifrons [E. Linares \& A. Galán 4546 (USP 3382)], E: T. fernandezianum [E. Linares, J. Montoya \& A. Galán 4537 (USP 3389)], F: T. spathulatum [E. Linares \& A. Galán 4535c (USP 3393)], G: T. argutifrons [O. Rangel, J. Pinto \& A. Galán 4549 (USP 3405)], H: T. craspedotoides [O. Rangel, J. Pinto \& A. Galán 4548 (USP 3396)].

Figure 2. Achenes of the studied Taraxacum species (bars $=1 \mathrm{~mm}$ ).

y Doll (1976), trataran de incluir otras especies. En Ecuador ya se reconoció la posible presencia de $T$. fernandezianum A.J. Richards aunque sin precisar referencias a pliegos de herbario (Jørgensen, 1999), y en Bolivia la de T. subspathulatum A.J. Richards (Jørgensen et al., 2016). En Colombia, se citan T. argutifrons A.J. Richards, T. craspedotoides A.J. Richards, T. officinale y T. spathulatum A.J. Richards (Bernal et al., 2017), mientras que en Chile T. angustisquameum Dahlst. ex H. Lindb., T. copidophyllum Dahlst., T. cuzcense A.J. Richards, T. fernandezianum, T. gilliesii Hook. \& Arn., T. marklundii Palmgr., T. officinale, T. patagonicum Uhlemann, T. plumbeum Dahlst. y $T$. vastisectum Markl., y en Argentina T. fernandezianum, $T$. gilliesii, T. laevigatum (Willd.) DC., T. officinale, y T. raunkiaeri Wiinst. in Raunkiaer (Freire, 2008, Urtebey \& Freire, 2015), basadas sobre todo en los trabajos de Richards (1976) y Uhlemann (2002).

Las nuevas colectas realizadas en Argentina, Colombia y Perú, que ordenamos por secciones según Kilian et al. (2016), dan a conocer nuevos registros para la flora de estos países, de los que se aportan datos sobre su distribución, ecología y fitosociología.. Las figuras 1 y 2 muestran la silueta de las hojas y la estructura de los aquenios respectivamente. Las fotografías de la figura 3 ilustran el hábito y capítulos de algunas de las especies.

Sect. Antarctica Hand.-Mazz., Monogr. Gatt. Taraxacum: XI (1907)

1. T. gilliesii Hook. \& Arn. in Companion Bot. Mag. 1: 31 (1835)
Lectotypus: Designado por Richards in Rhodora 78: 702 (1976): K 000222226

T. andinum Dahlst. in Arkiv Bot. 6(12): 12 (1907)

T. magellanicum Comm. ex Sch. Bip. in Flora 38: 122 (1855)

T. melanocarpum Hand.-Mazz., Monogr. Gatt. Taraxacum: 54 (1907)

Nuevas citas: ARGENTINA. Tierra del Fuego: Ushuaia, glaciar Martial, en pastos húmedos, 54²7'26.95"'S-68²3'37.64'”, $610 \mathrm{~m}$, 26/01/2016, E. Linares \& A. Galán 4547a (CPUN), E. Linares \& A. Galán 4547b (MA), E. Linares \& A. Galán 4547c (MO, USP 3399), E. Linares \& A. Galán 4556 (USP 3400).

Observaciones: Aunque esta especie ya fue citada por numerosos autores en Tierra del Fuego (Freire, 2008), no hemos encontrado ningún registro próximo a nuestra localidad. El material de T. melanocarpum al que hace referencia Herrera (1941) en Cusco, Perú (CUZ 020413) debe llevarse a $T$. fernandezianum.

Ecología y fitosociología: Su ecología se restringe a los pastizales húmedos de Festuca pallescens (St.-Yves) Parodi de la Patagonia, pertenecientes a la alianza Carico sorianoiFestucion pallescentis Roig et al. 1985.

Sect. Erythrosperma $(H$. Lindb.) Dahlst. in Acta FI. Sueciae 1: 36 (1921)

2. T. plumbeum Dahlst. in Ark. Bot. 10(6): 2 (1911) Lectotypus: Designado por Doll in Feddes Repert. 84: 123 (1973): S 10-37719

Nuevas citas: ARGENTINA. Santa Cruz, 

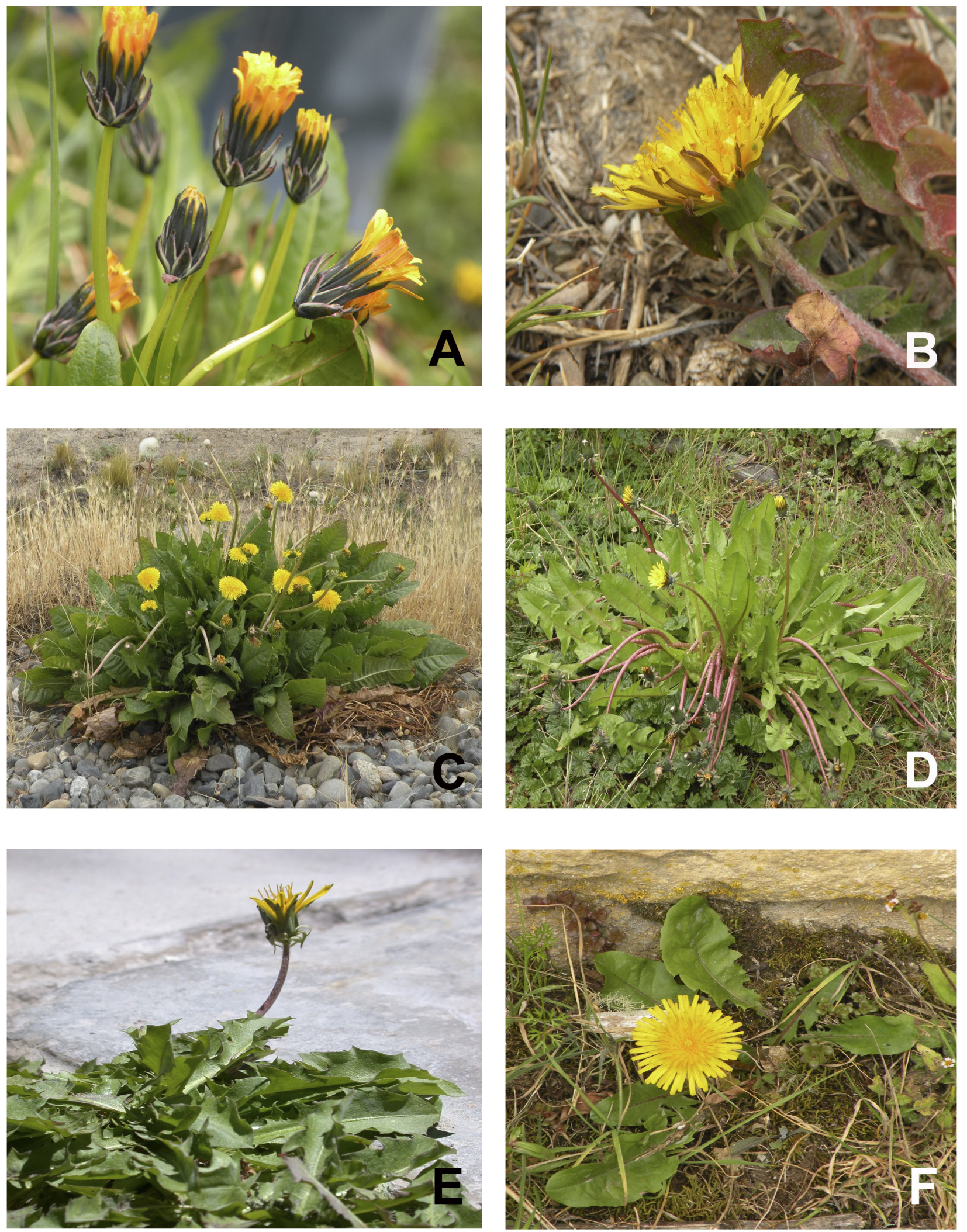

Figura 3. Hábito y capítulos de algunas especies estudiadas. A: T. gilliesii (Ushuaia, Argentina), B: T. plumbeum (El Calafate, Argentina), C: T. stictophyllum (El Calafate, Argentina), D: T. duplidentifrons (Ushuaia, Argentina), E: T. fernandezianum (Sondor, Perú), 1: T. spathulatum (Kuelap, Perú).

Figure 3. Habit and heads of some studied species. 
El Calafate, estancia al sur de la población, hacia la quebrada, en pastizales secos, 50²3'11.11'S-72¹3'19.77'W, 475 m, 24/1/2016, E. Linares \& A. Galán 4524a (CPUN), E. Linares \& A. Galán 4524b (MA), E. Linares \& A. Galán 4524c y $d$ (USP 3398); Estancia Anita junto al río Mitre, en pastizales secos, $50^{\circ} 27^{\prime} 09.33^{\prime \prime} S-72^{\circ} 34^{\prime} 02.27^{\prime \prime} \mathrm{W}$, $318 \mathrm{~m}, 23 / 1 / 2016$, E. Linares \& A. Galán 4525 (USP 3397).

Observaciones: Se trata de los primeros registros de esta especie para Argentina (Freire, 2008).

Ecología y fitosociología: Habita en suelos bastante secos con pastizales pisoteados de escasa altura, con Acaena splendens Hook. \& Arn., entre los matorrales de Mulinum spinosum Pers. de la alianza Stipo-Nassauvion ulicinae Roig et al. 1985.

Sect. Naevosa M.P. Christ. in Rosenvinge \& Warming, Bot. Iceland 3: 303 (1942)

3. T. stictophyllum Dahlst. in Ark. Bot. 12(2): 38 (1912)

Lectotypus: Designado por Lundevall \& Øllgaard in Preslia 71: 149 (1999): S 11-21617

Nuevas citas: ARGENTINA. Santa Cruz: EI Calafate, junto al hotel Los Gnomos, en pastizales ruderales, 50²0'29.71''S-72 ${ }^{\circ} 14^{\prime} 51.58^{\prime \prime} \mathrm{W}, 227 \mathrm{~m}$, 24/1/2016, E. Linares \& A. Galán 4522a (MO), E. Linares \& A. Galán 4522b (MA), E. Linares \& A. Galán 4522c-e (USP 3379); El Calafate, estancia al sur de la población, hacia la quebrada, en pastizales secos, $50^{\circ} 23^{\prime} 11.11^{\prime \prime} S-72^{\circ} 13^{\prime} 19.77^{\prime \prime W}$, $475 \mathrm{~m}$, 24/1/2016, E. Linares \& A. Galán 4523 (USP 3380).

Observaciones: Estas son las primeras citas para América del Sur de esta especie del norte de Europa (Gran Bretaña, Islas Feroe, Islandia y Noruega) (Richards \& Sell, 1984). La hacen inconfundible sus hojas ásperas y pelosas, el pecíolo áptero, el nervio central y pecíolo purpúreos, y el lóbulo terminal de un tamaño mucho mayor que los laterales, y con grandes dientes.

Ecología y fitosociología: Se muestra ruderal en comunidades de Hordeum murinum L. de la clase Stellarietea mediae Tüxen et al. 1951, junto a los núcleos poblacionales.

4. T. duplidentifrons Dahlst. in Johnston, Add. Fl. Orkney 11: 16 (1928)

Lectotypus: Designado por Haworth \& Richards in Watsonia 18: 127 (1990): E 00279018 T. raunkiaeri Wiinst. in Raunkiaer, Dansk Exkurs.-Fl., ed. 5: 303 (1934)

Nuevas citas: ARGENTINA. Tierra del
Fuego: Ushuaia, glaciar Martial, borde del bosque de Nothofagus betuloides, $54^{\circ} 47^{\prime} 31.39$ "S68'23'20.40'W, 554 m, 26/1/2016, E. Linares \& A. Galán 4544 (MA), E. Linares \& A. Galán 4545 (MO), E. Linares \& A. Galán 4543 (USP 3381), 4546 (USP 3382).

Observaciones: Es una especie ampliamente repartida por el norte de Europa (Richards \& Sell, 1984) que también se encuentra como adventicia en otras partes del mundo como Madeira (Press, 1994), Azores y Canarias (Galán de Mera et al., 2017), donde la podemos diferenciar por sus pecíolos alados. Fue citada en Estancia Harberton (Argentina) por Richards (1976), a más de $80 \mathrm{Km}$ de nuestra localidad.

Ecología y fitosociología: Crece en los bordes de caminos y pastos adyacentes a los bosques patagónicos de Nothofagus betuloides (Mirb.) Oerst. de la alianza Embothrio-Nothofagion betuloidis Roig et al. 1985.

Sect. Mexicana A.J. Richards in Rhodora 78: 684 (1976)

5. T. fernandezianum Dahlst. in Skottsberg, Nat. Hist. Juan Fernández 2: 226 (1922)

Lectotypus: Designado por Richards in Rhodora 78: 691 (1976): S R-6059

T. subspathulatum A.J. Richards in Rhodora 78: 692 (1976)

T. andiniforme Doll in Feddes Repert. 87: 447 (1976)

T. cuzcense A.J. Richards in Rhodora 78: 697 (1976)

T. melanocarpum sensu Herrera, Sin. FI. Cuzco I: 437 (1941)

Nuevas citas: ARGENTINA. Entre Ríos: Diamante, en céspedes con Cynodon dactylon, 32'04'03.71"'S-60³8'26.64'W, 73 m, 30/01/2016, E. Linares \& A. Galán 4538 (MO), E. Linares \& A. Galán 4539 (MO), 4540 (MA), E. Linares \& A. Galán 4541 (USP 3383), 4542 (USP 3408). PERÚ. Amazonas: Ocol, 6015'05.46"S-77³4'13.48'W, $2378 \mathrm{~m}$, 08/08//2016, E. Linares, J. Montoya \& A. Galán 4536 (USP 3388); Ocol, borde de carretera

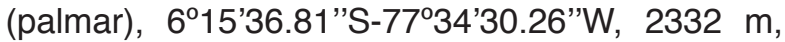
08/08/2016, E. Linares, J. Montoya \& A. Galán 4537 (USP 3389). Arequipa: Sondor, huerta, 15'15'41.11"S-73³0'11.42"W, 01/01/2016, E. Linares \& A. Galán 4527 (CPUN), E. Linares \& A. Galán 4528 (MA), E. Linares \& A. Galán 4529 (USP 3386), 4530 (USP 3384), 4531 (USP 3385). Cajamarca: El Empalme, en la jalca, 642'54.84"S78 38'28.91''W, 3717 m, 03/08/2016, E. Linares, J. Montoya \& A. Galán 4532 (CPUN), E. Linares, J. Montoya \& A. Galán 4533 (USP 3390). Puno: entre 
Antauta y San Isidro (Condorire), en la cuneta de la carretera, 14²2'29.48'S-70²1'42.82'W, 4058 $\mathrm{m}, 11 / 01 / 2012$, E. Linares \& A. Galán 2659 (USP 3228). De Macusani a Ollachea, 1343'22.80"S70²3'49.16'W, $4210 \mathrm{~m}, 03 / 01 / 2015$, E. Linares \& A. Galán 4526 (USP 3387).

Observaciones: Esta especie, cuyo material tipo procede de las islas Juan Fernández (Chile), parece ser la que presenta una distribución más amplia en América del Sur, donde posiblemente es adventicia (Richards, 1976); al menos, no se conoce su uso por las culturas pre-hispánicas (Chávez Velásquez, 1977). Se caracteriza por sus hojas dentadas frecuentemente con lóbulos poco definidos y el nervio principal purpúreo, y los aquenios con el cono subcilíndrico, caracteres que se repiten tanto en el holótipo de $T$. subspathulatum (Tucumán, Argentina, US 00119861), como en el de T. cuzcense (Santiago, Chile, US 00119853). El único carácter que podría separar a $T$. cuzcense es el hábito más pequeño de las plantas debido a la altitud a la que viven (3000-4050 m); incluso la forma del lóbulo terminal en poblaciones de los alrededores del Cusco y de Bolivia se confunde con la de T. fernandezianum. Algo similar sucede con T. andiniforme (holótipo: [Perú] La Oroya-Huánuco, JE 00001152), pues la forma del lóbulo terminal de sus hojas dentadas sigue el mismo esquema que en el T. fernandezianum determinado por Richards en La Oroya (US 1044255) y Huánuco (US 1999863). El capítulo desecado y más obscuro de las pequeñas plantas del Cusco conservadas en CUZ (020413) y US (761629) posiblemente fue lo que llevó a F. Herrera y a C. Vargas a considerar el nombre de T. melanocarpum $(=T$. gilliesii). Del material estudiado, las citas de Entre Ríos (Argentina), y Amazonas y Cajamarca (Perú) son nuevas localizaciones al nivel provincial y departamental respectivamente tras los datos de Freire (2008) en la provincias de Neuquén y Salta (Argentina) y de Galán de Mera \& Linares Perea (2008) en los departamentos del centro y sur del Perú. Los especímenes determinados en este último trabajo como T. ekmanii Dahlst. deben llevarse a $T$. fernandezianum, pues aunque son plantas de gran tamaño, tienen caracteres de la sección Mexicana.

Ecología y fitosociología: T. fernandezianum tiene preferencia por distintos hábitats de la puna, tanto por los pajonales del orden Calamagrostietalia vicunarum Rivas-Martínez \& Tovar 1982 como por los suelos removidos de los cultivos con comunidades herbáceas pertenecientes al orden Calandrinietalia ciliatae Galán de Mera et al. 2011. También lo encontramos en el orden paramuno Agrostio tolucensis-Paspaletalia humboldtiani
Galán de Mera et al. 2015 en el entorno de la depresión de Huancabamba (Cajamarca, Perú).

6. T. spathulatum A.J. Richards in Rhodora 78: 694 (1976)

Holotypus: México: Oaxaca, Distrito del Centro, Acueducto de Xochimilco, $1570 \mathrm{~m}$, 16.III.1937, C. Conzatti 5259 (US 0119860)

Nuevas citas: COLOMBIA. Cundinamarca: EI Encenillo, en el interior de bosque de Weinmannia tomentosa, 452'18.01'N-7351'15.58'O, 3084 m, 04/11/2016, O. Rangel, J. Pinto \& A. Galán 4550 (USP 3391). PERÚ. Amazonas: Ocol, 6¹1'25.42'S-78³9'57.47'O, 2480 m, 08/08/2016, E. Linares, J. Montoya \& A. Galán 4534 (USP 3392); Kuelap, 6²5'17.61"S-7755'29"O, 2901 m, 09/08/2016, E. Linares, J. Montoya \& A. Galán (USP-foto). Cajamarca: campus de la Universidad Nacional de Cajamarca, en acequia, 7010'01.64"S78²9'47.42'O, 2684 m, 12/08/2016, E. Linares \& A. Galán 4535a (CPUN), E. Linares \& A. Galán 4535 b-c (USP 3393).

Observaciones: Se trata de los primeros registros de esta especie en el Perú tras la cita de Richards (1976) en Putumayo (Colombia). Las plantas colectadas en Colombia son una novedad departamental.

Ecología y fitosociología: La proximidad biogeográfica entre los Andes orientales del departamento de Amazonas (Perú) y los bosques húmedos colombianos de la alianza Weinmannion tomentosae Cuatrecasas 1934 se pone de manifiesto con los suelos obscuros donde crece T. spathulatum como especie ruderal de la clase tropical Soncho-Bidentetea pilosi Hoff in Hoff \& Brisse 1983.

7. T. argutifrons A.J. Richards in Rhodora 78: 694 (1976)

Holotypus: Mexico: Charcas, San Luis Potosi, 13.VII.1904, E. Palmer 180 (US00119849)

Nuevas citas: COLOMBIA. Cundinamarca: Guasca, El Encenillo, en prados junto a bosque de Weinmannia tomentosa, 501'27.98"N7350'12.09'W, 3084 m, 04/11/2016, O. Rangel, J. Pinto \& A. Galán 4549 (USP 3405, 3406).

Observaciones: Segunda cita colombiana tras la de Putumayo (Richards, 1976).

Ecología y fitosociología: Ampliamente repartida por Centroamérica, encuentra en Colombia su distribución más meridional (Richards 1976), en áreas que han sido cultivadas, y cuya vegetación es de la clase Soncho-Bidentetea pilosi Hoff in Hoff \& Brisse 1983.

8. T. craspedotoides A.J. Richards in Rhodora 78: 697 (1976) 
Holotypus: Venezuela: Entre La Venta y Laguna Huacha, 3450-3650 m, 21.XI.1959, H.G. Barclay \& P. Juajibioy 9862 (US 00119852)

\section{Material estudiado: COLOMBIA.} Cundinamarca: Guasca, en céspedes de la población, 446'18.10'N-7351'03.92"O, $2664 \mathrm{~m}$, 04/11/2016, O. Rangel, J. Pinto \& A. Galán 4548 (USP 3396), O. Rangel, J. Pinto \& A. Galán 4553 (MA); El Encenillo, en bosque de Weinmannia tomentosa, 452'18.01"N-7351'15.58"O, $3084 \mathrm{~m}$, 04/11/2016, O. Rangel, J. Pinto \& A. Galán 4551 (USP 3404), O. Rangel, J. Pinto \& A. Galán 4552 (USP 3395); páramo de Guasca, 4 ${ }^{\circ} 58^{\prime} 46.66^{\prime \prime} \mathrm{N}$ 734'13.11"O, $3345 \mathrm{~m}, 04 / 11 / 2016$, O. Rangel, J. Pinto \& A. Galán 4554 (MA), O. Rangel, J. Pinto \& A. Galán 4555 (USP 3394).

Observaciones: Se trata de un nuevo registro tras la cita del departamento de Caldas (Parque Nacional Los Nevados) (Bernal et al., 2017). Al igual que en Venezuela, en Colombia esta especie alcanza el páramo, diferenciándose por sus hojas de lóbulos laterales definidos, el terminal sagitado, y el nervio principal blanquecino.

Ecología y fitosociología: Se distribuye en el páramo de Venezuela y Colombia entre plantas de la clase Pentacalio vaccinioidis-Calamagrostietea effusae Rangel \& Pinto-Zárate in Pinto-Zárate \& Rangel 2010.

\section{Agradecimientos}

Deseamos expresar nuestro agradecimiento a la guardianía de la Reserva Biológica El Encenillo (Guasca, Cundinamarca, Colombia); al Centro de Investigaciones Científicas y Transferencia de Tecnología a la Producción de Entre Ríos (Argentina), y al Prof. Gustavo Iberico, director del Herbario CPUN de la Universidad Nacional de Cajamarca (Perú) por facilitarnos las colectas y su conservación. Gracias a los Profs. Orlando Rangel y Jairo Pinto pudimos colectar especímenes en el páramo de Guasca. A Julio Pinto por su apoyo y guía por los caminos del Perú.

\section{Bibliografía}

Beltrán, H. (2016). Las Asteráceas (Compositae) del distrito de Laraos (Yauyos, Lima, Perú. Revista peruana de biología, 23(2), 195-220.

doi: http://dx.doi.org/10.15381/rpb.v23i2.12439

Bernal, R., Gradstein, S.R. \& Celis, M. (2017). Catálogo de plantas y líquenes de Colombia (fecha de consulta: 08/09/2017). Bogotá: Universidad Nacional de Colombia. http://catalogoplantasdecolombia.unal.edu. co.

Brako, L. \& Zarucchi, J.L. (1993). Catálogo de las Angiospermas y Gimnospermas del Perú. St. Louis, MO: Missouri Botanical Garden.

Chávez Velásquez, N.A. (1977). La Materia Médica en el
Incanato. Lima: Mejía Baca.

Doll, R. (1976). Eine neue Taraxacum-Sippe aus Peru. Feddes Repertorium, 87, 447-448. doi: 10.1002/ fedr. 19760870702

Freire, S.E. (2008). Asteraceae. In: F.O. Zuloaga, O. Morrone \& M.J. Belgrano (Eds.), Catálogo de las Plantas Vasculares del Cono Sur, Vol. 2 (pp. 11541564). St. Louis, MO: Missouri Botanical Garden Press.

Galán de Mera, A. (2017). Taraxacum F.H. Wigg. In S. Castroviejo et al. (Eds.), Flora iberica Vol. 16(2) (pp. 963-1062). Madrid: Consejo Superior de Investigaciones Científicas.

Galán de Mera, A. \& Linares Perea, E. (2008). Taraxacum officinale F.H. Wigg. (Asteraceae) no es una especie peruana. Arnaldoa, 15(1), 41-44.

Galán de Mera, A., Linares Perea, E. \& Vicente Orellana, J.A. (2017). Taraxacum (Asteraceae) in the Azores, Madeira and the Canary Islands. Annales Botanici Fennici, 54, 273-285.

Handel-Mazzetti, H. (1907). Monographie der Gattung Taraxacum. Leipzig und Wien: Franz Deuticke.

Herrera, F.L. (1941). Sinopsis de la Flora del Cusco. Tomo I. Parte Sistemática. Lima: Edición del autor.

Jørgensen, P.M. (1999). Taraxacum. In: P.M. Jørgensen \& S. León-Yáñez (Eds.), Catalogue of the Vascular Plants of Ecuador (p. 311). St. Louis, MO: Missouri Botanical Garden Press.

Jørgensen, P.M., Nee, M.H. \& Beck, S.G. (2016). Bolivia Catalogue (fecha de consulta 06/07/2017). St: Louis, MO: Missouri Botanical Garden. http://www.tropicos. org/.

Kilian, N., Hand, R. \& Raab-Straube, E. (2017). Cichorieae Systematics Portal (fecha de consulta: 10/09/2017). http://cichorieae.e-taxonomy.net/portal/.

Press, J.R. (1994). Taraxacum F.H. Wigg. In J.R. Press \& M.J. Short (Eds.), Flora of Madeira (pp. 378-379). London: HMSO.

Richards, A.J. (1976). An account of some neotropical Taraxacum species. Rhodora, 78, 682-706.

Richards, A.J. \& Sell, P.D. (1984). Taraxacum. In T.G. Tutin, V.H. Heywood, N.A. Burges, D.M. Moore, D.H. Valentine, S.M. Walters \& D.A. Webb (Eds.), Flora Europaea, Vol. 4 (pp.332-343). Cambridge: Cambridge University Press.

Uhlemann, I. (2002). A new species of the genus Taraxacum F.H. Wigg. (Asteraceae, Lactuceae) from South America. Feddes Repertorium, 113, 329-334. doi: $10.1002 / 1522-239 X(200210) 113: 5 / 6<329:: A I D-$ FEDR329>3.0.CO;2-8

Uhlemann, I., Kirschner, J. \& Štěpánek, J. (2004). The Genus Taraxacum (Asteraceae) in the Southern Hemisphere. I. The Section Antarctica HandelMazzetti and Notes on Dandelions of Australasia. Folia Geobotanica, 39, 205-220.

Urtebey, E. \& Freire, S.E. (2015). Tribu Cichorieae. In F.O. Zuloaga, M.J. Belgrano \& A.M. Anton (Eds.), Flora Vascular de la República Argentina, Vol. 7(2): Dicotyledoneae-Asteraceae (Cichorieae, HeleniaeMutisieae) (pp. 6-83). Buenos Aires: Instituto de Botánica Darwinion.

Van Baarlen, P., van Dijk, P.J., Hoekstra, R.F. \& De 
Jong, J.H. (2000). Meiotic recombination in sexual diploid and apomictic triploid dandelions (Taraxacum officinale L.). Genome, 43, 827-835. doi: 10.1139/g00047.
Vašut, R.J. (2003). Taraxacum sect. Erythrosperma in Moravia (Czech Republic): Taxonomic notes and the distribution of previously described species. Preslia, 75, 311-338. 